\title{
Identification of novel genetic etiology and key molecular pathways for seminoma via network-based studies
}

\author{
YUANLEI CHEN $^{1 *}, \mathrm{CHAO} \mathrm{QI}^{2^{*}}, \mathrm{LIQUN}^{\mathrm{XIA}}{ }^{1^{*}}$ and GONGHUI LI${ }^{1}$ \\ ${ }^{1}$ Department of Urology and Chawnshang Chang Liver Cancer Center and ${ }^{2}$ Department of Clinical Laboratory, \\ Sir Run Run Shaw Hospital, Zhejiang University, Hangzhou, Zhejiang 310016, P.R. China
}

Received May 22, 2017; Accepted July 19, 2017

DOI: $10.3892 /$ ijo.2017.4092

\begin{abstract}
Seminoma is the most common testicular malignant germ cell tumor which severely threaten male reproductive health. However, the pathogenesis and progression mechanisms are still unclear and little research is carried out to uncover the mechanism of this cancer. To identify gene signatures that are expected to converge on particular molecular pathways may drive the development of the core cancer characteristics in seminoma. We downloaded the gene expression profiles GSE18155 from GEO database and obtained the differentially expressed genes (DEGs). Then, we performed Gene ontology (GO) and Kyoto Encyclopedia of Genes and Genomes pathway (KEGG) enrichment analyses
\end{abstract}

Correspondence to: Professor Gonghui Li, Department of Urology and Chawnshang Chang Liver Cancer Center, Sir Run Run Shaw Hospital, Zhejiang University, Hangzhou, Zhejiang 310016, P.R. China E-mail: ligonghui1970@hotmail.com

*Contributed equally

Abbreviations: GEO, Gene Expression Omnibus; DEGs, different expression genes; KEEG, Kyoto Encyclopedia of Genes and Genomes; STRING, Search Tool the Retrieval of Interacting Genes; GO, Gene ontology; PPI, protein-protein interaction; IHC, immunohistochemistry; RT-qPCR, reverse transcription-polymerase chain reaction; TGCTs, testicular germ cell tumors; NSGCTs, non-seminomatous germ cell tumors; AFP, $\alpha$-fetoprotein; HCG, human chorionic gonadotrophin; $\mathrm{LDH}$, lactate dehydrogenase; MF, molecular function; CC, cellular component; $\mathrm{BP}$, biological process; POU5F1, POU class 5 homeobox 1; NANGO, Nanog homeobox; LIN28, Lin-28 homolog; TFAP2C, transcription factor AP- $\gamma$; UBC, ubiquitin C; UBB, ubiquitin B; AR, androgen receptor; PTEN, phosphatase and tensin homolog; CDK, cyclin-dependent kinases; AKT, RAC- $\alpha$ serine/threonine-protein kinase; MAPK8, mitogenactivated protein kinase 8; TOP2A, topoisomerase (DNA) II $\alpha$; CDKN1A, cyclin-dependent kinase inhibitor 1a; RAC1, Ras-related C3 botulinum toxin substrate 1; UPS, ubiquitin-proteasome system; WNT, wingless-type; PI3K, phosphatidylinositol-4,5-bisphosphate 3-kinase

Key words: genetic etiology, bioinformatics analysis, seminoma, microarray, molecular pathways, protein-protein interaction via DAVID. In total, 3,502 DEGs were identified, including 1,563 upregulated genes and 1,939 downregulated genes. The top 10 hub genes, $U b c, U b b, M a p k 8, A r$, Pten, $C d k 2, C d k 4$, Racl, Top $2 a$ and $C d k n l a$ were identified from the proteinprotein interaction (PPI) network which was constructed by Cytoscape. Sub-networks analyzed by MCODE plugin revealed these genes were involved in significant molecular pathways, including ERBB2 signaling pathway, pathways in cancer, PI3K-AKT signaling pathway and cell cycle. Finally, validation of hub genes was checked via reverse transcription-polymerase chain reaction (RT-PCR) assay and immunohistochemistry (IHC). In conclusion, our study revealed that the identified hub genes increased the understanding of genetic etiology and molecular mechanisms underlying the development of seminoma, in return, they could be used as potential diagnostic biomarkers and therapeutic molecular targets for seminoma.

\section{Introduction}

Testicular germ cell tumors (TGCTs) are the most common solid tumors among adolescent and young adult male, of which the incidence rates is actually increasing every year (1). Seminoma is the most common testicular germ cell tumor which often develops in the cryptorchid testis and occurs between 35 and 45 years (2). As we know, seminoma, like other cancers, is considered as a histologically heterogeneous disease in which gene aberrations have a prominent role in cancer occurrence, progression, and metastasis $(3,4)$. Emerging evidence has revealed that multiple genes and molecular pathways participate in the initiation and progression of human cancers. Targeting of hub genes and key molecular pathways has been recognized as a promising approach in the discovery and treatment of cancer (5). However, only few aspects of the mechanism and gene expression of this cancer are well studied and remain largely unclear. Therefore, to get a better understanding of the genetic etiology and molecular mechanism involved in the occurrence, progression, and metastasis of seminoma is extremely vital for acquiring more effective diagnostic biomarkers and therapeutic strategies.

The conventional serum diagnostic markers, such as $\alpha$-fetoprotein (AFP), human chorionic gonadotrophin (hCG) and lactate dehydrogenase (LDH), show some utility in the diagnosis and follow-up purposes of TGCT (6). However, AFP 
and hCG also exhibit certain limited sensitivity and specificity, being indicative of yolk sac tumor (AFP) and choriocarcinoma or syncytiotrophoblast (hCG) subtypes. Furthermore, LDH is recognized as a very nonspecific biomarker. Therefore, seminomas and non-seminomatous GCTs (NSGCTs) comprising a pure embryonal carcinoma subtype are often negative for these conventional markers (7-9).

Accumulated studies have shed light on the transformation of normal gonocytes into malignant germ cell tumors (Fig. 1). Gain of chromosome arm 12p is considered to have a highest effect and be nearly universal in TGCTs $(10,11)$. Despite germline genome wide association studies have confirmed several risk loci for TGCTs, only Kit and Ras gene family have been implicated repeatedly in different TGCTs (12). Besides, genome-wide microarray screens have identified some elevated expression of embryonic pluripotency-related genes such as Pou5f1, Lin28, Nanog and Tfap2c in germ cell neoplasia in situ, embryonal carcinoma and seminoma (4). Despite these former efforts to discover genetic foci of susceptibility, no validated molecular biomarkers exist that can be used for precise screening, diagnostic or therapeutic purposes.

In this study, we focused on the most common testicular germ cell tumors - seminoma (roughly $56 \%$ of cases, peak incidence at 35 years (13). We used a systematic approach that can be used for acquiring novel molecular biomarkers for seminoma. Based on data from GEO online database, we explored the differently expressed genes (DEGs), related molecular pathways and consequently constructed a regulatory network. Then the top 10 hub genes were chosen and determined by RT-qPCR and IHC assay, which could be used as potential biomarkers for diagnosis and may also be related with prognosis. Furthermore, these targets could possibly also give us a novel insight into seminoma pathogenesis.

\section{Materials and methods}

Microarray data. The gene expression profiles of GSE18155, including 12 seminoma samples and 5 normal testis samples, were obtained from National Center of Biotechnology Information (NCBI) GEO database (GEO, http://www.ncbi. nlm.nih.gov/geo/). The GSE18155, which was based on GPL96 [HG-U133A] Affymetrix Human Genome U133A array, was submitted to the database by Matthew Jonathan Murray et al.

Differentially expressed gene analysis. The raw data were analyzed by using GCBI online software (https://www.gcbi. com.cn/gclib/html/index). Upregulated and downregulated genes were identified between seminoma and normal controls. A classical criteria of t-test was used to identify DEGs with a change $\geq 2$-fold and defined a P-value cutoff $<0.05$ to be statistically significant.

Functional and pathway enrichment analysis of DEGs. It is well known that Database for Annotation Visualization and Integrated Discovery (DAVID) is a common useful method to perform Gene ontology analysis (GO) and Kyoto Encyclopedia of Genes and Genomes (KEGG). We chose the human genome as the background list and human was used as the species. $\mathrm{P}<0.05$ was considered statistically significant.
PPI network construction and selection of modules. We used online tool Search for the Retrieval of Interacting Genes (STRING) database (http://www.string-db.org) to evaluate the protein-protein interaction (PPI) information. To identify the interactive relationship among DEGs, we imported the DEGs to STRING and only experimentally validated interactions with a combined score $>0.7$ was selected as significant. Then these significant DEGs were mapped into Cytoscape plugin to create network visualizations. Finally, we put the resulting PPI network to module analysis with the Plugin MCODE with the default parameters (Degree cutoff $\geq 2$, Node score cutoff $\geq 2$, $\mathrm{K}$-core $\geq 2$, and Max depth=100). Moreover, the function and pathway enrichment analysis were performed for DEGs in the modules. $\mathrm{P}<0.05$ was considered to be significant.

Analysis of hub gene mRNA and protein expression in human seminoma. Top 5 hub gene protein expression in seminoma tissues and normal tissues was determined from the human protein atlas (www.proteinatlas.org). Meanwhile, mRNA expression was determined via analysis of Korkola Seminoma Statistics, which are available through Oncomine (Compendia Biosciences, www.oncomine.org). High and low groups were defined as above and below the mean, respectively.

Clinical specimens. Five tumor samples and paired adjacent non-cancerous tissues were obtained from 5 seminoma patients subjected to orchiectomy in the Sir Run Run Hospital of Zhejiang University who were diagnosed with seminoma by more than two pathologists.

Quantitative real-time PCR ( $q R T-P C R)$. Total RNA was extracted from patient tissues using TRIzol reagent (Invitrogen, USA) and was stored at $-80^{\circ} \mathrm{C}$ until use. Reverse Transcription system (Promega) was used for cDNA synthesis according to the manufacturer's protocol. The mRNA expression levels of hub genes were measured by quantitative real-time PCR using the ABI PRISM 7500 Sequence Detector system (Applied Biosystems, USA), and was normalized to an internal standard (glyceraldehyde-3-phosphate dehydrogenase, GAPDH). PCR primer used were as follows: AR: forward, 5'-TACCG CATGCACAAGTCCCG-3'; reverse, 5'-TCACTGGGTGTGG AAATAGA-3'. UBB: forward, 5'-GGTGAGCTTGTTTGTGT CCCTGT-3'; reverse, 5'-TCCACCTCAAGGGTGATGGTC-3'. UBC: forward, 5'-TGCACCTGGTACTCCGTC TCA-3'; reverse, 5'-CAGTGAGTGTCTTCACGAAGATTTG-3'. CDK4: forward, 5'-ATGGCTACCTCTCGATATG AGC-3'; reverse, 5'-CATTGGGGACTCTCACACTCT-3'. PTEN: forward, 5'-ACCCACACGACGGGAAGACA-3'; reverse, 5'-CTG TTTGTGGAAGAACTCTACTTTGATAT CAC-3'. GAPDH: forward, 5'-AGACAGCCGCATCTTCTTGT-3'; reverse, 5'-TGA TGGCAACAATGTCCACT-3'. The reaction protocol involved heating for $3 \mathrm{~min}$ at $95^{\circ} \mathrm{C}$, followed by 45 cycles of amplification $\left(15 \mathrm{sec}\right.$ at $95^{\circ} \mathrm{C}$ and $1 \mathrm{~min}$ at $\left.60^{\circ} \mathrm{C}\right)$. All reactions were done in triplicate.

\section{Results}

Identification of DEGs in seminoma. In order to reveal the different expression of genes involved in seminoma, we selected the publicly available microarray datasets (12 seminoma 


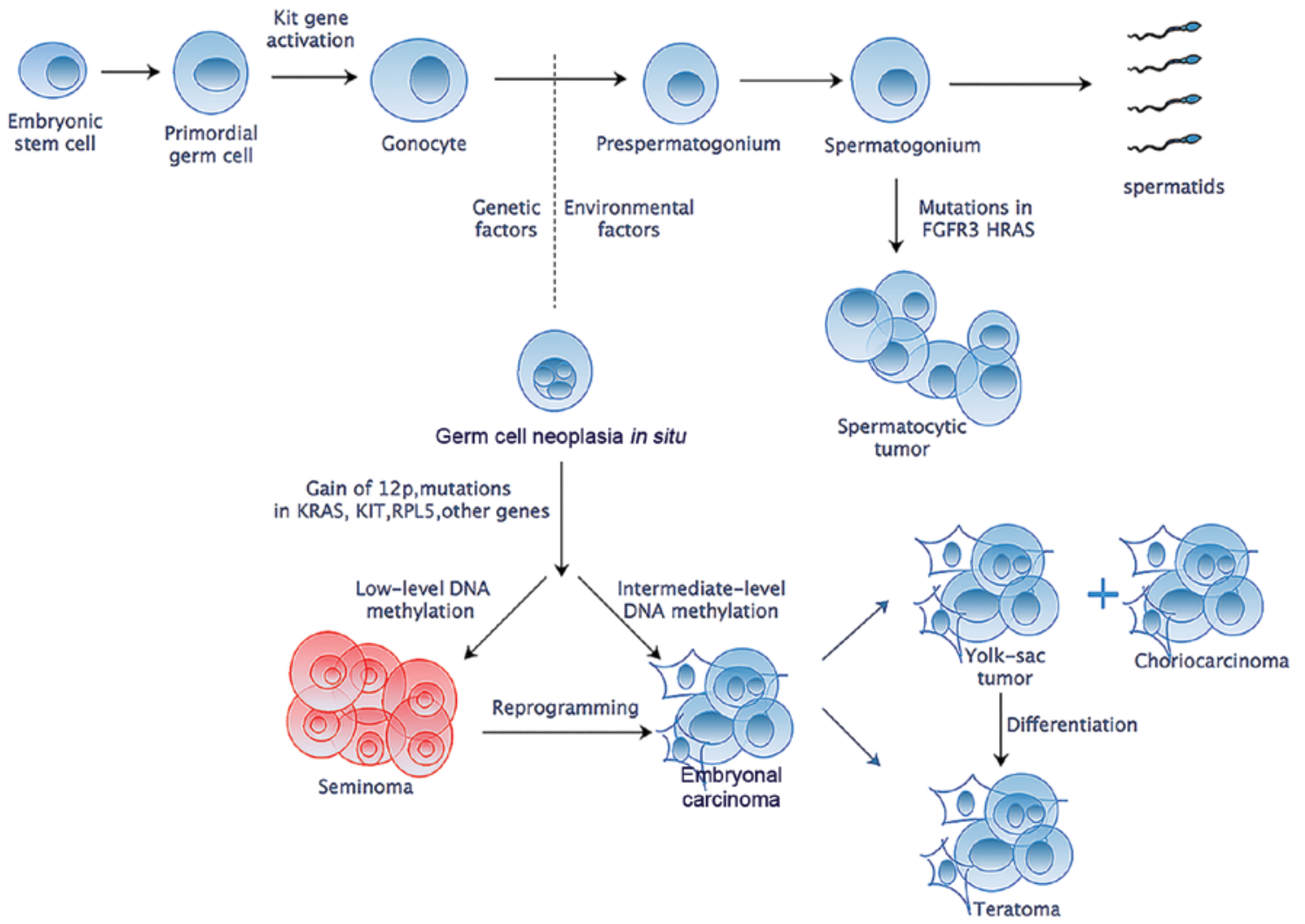

Figure 1. Traditional genetic aberrations and susceptibility genes related to the pathogenesis of testicular germ cell tumors.

A

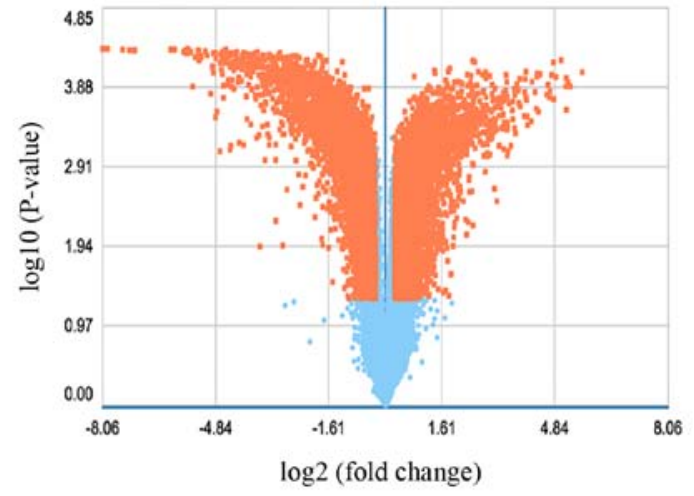

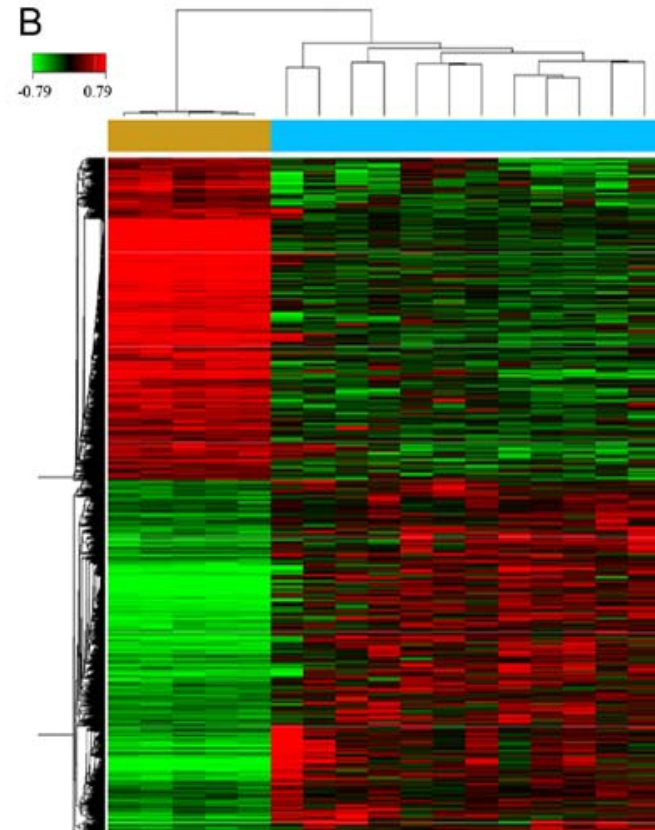

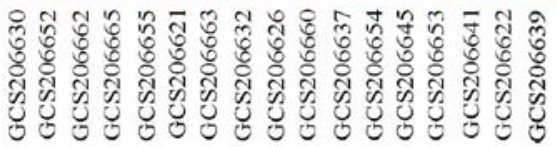

Figure 2. Volcano plot of the differentially expressed genes. Heat-map overview of the differentially expressed genes. Seminoma samples versus control samples. Red, upregulation. Green, downregulation.

samples and 5 normal samples) from the GEO database and used the GCBI analysis to identify DEGs between them. With the criteria $\mathrm{P}<0.05$ and fold control $(\mathrm{FC}) \geq 2$, a total of 3,502 genes were identified after the analysis of GSE18155, among which, 1,563 were upregulated and 1,939 were downregulated. DEGs expression volcano plot and heat map are presented in Fig. 2. 
Table I. Gene ontology analysis of differentially expressed genes associated with seminoma.

\begin{tabular}{lllcr}
\hline Expression & \multicolumn{1}{c}{ Category } & \multicolumn{1}{c}{ Go term } & Gene count \% & P-value \\
\hline Upregulated & GOTERM_BP_FAT & Immune response & 23.3 & $2.0 \mathrm{E}-57$ \\
& GOTERM_BP_FAT & Cell adhesion & 19.1 & $3.3 \mathrm{E}-26$ \\
& GOTERM_BP_FAT & Cell proliferation & 18.2 & $1.1 \mathrm{E}-17$ \\
& GOTERM_BP_FAT & Cell migration & 13.0 & $5.3 \mathrm{E}-17$ \\
& GOTERM_CC_FAT & Cytoplasm & 36.5 & $1.1 \mathrm{E}-12$ \\
& GOTERM_CC_FAT & Plasma membrane & 27.4 & $7.5 \mathrm{E}-7$ \\
& GOTERM_CC_FAT & Extracellular exosome & 27.3 & $1.9 \mathrm{E}-31$ \\
& GOTERM_MF_FAT & Protein binding & 61.8 & $1.2 \mathrm{E}-25$ \\
& GOTERM_MF_FAT & PolyA RNA binding & 12.2 & $1.4 \mathrm{E}-15$ \\
& GOTERM_MF_FAT & Cadherin binding in cell adhesion & 4.30 & $1.4 \mathrm{E}-10$ \\
Downregulated & GOTERM_BP_FAT & Reproduction & 14.7 & $3.7 \mathrm{E}-26$ \\
& GOTERM_BP_FAT & Cell cycle & 13.9 & $9.8 \mathrm{E}-12$ \\
& GOTERM_BP_FAT & Spermatogenesis & 9.10 & $6.0 \mathrm{E}-40$ \\
& GOTERM_BP_FAT & Male gamete generation & 9.10 & $7.6 \mathrm{E}-40$ \\
& GOTERM_CC_FAT & Cytoplasm & 36.5 & $2.0 \mathrm{E}-19$ \\
& GOTERM_CC_FAT & Nucleus & 35.2 & $8.4 \mathrm{E}-12$ \\
& GOTERM_CC_FAT & Cytosol & 23.9 & $3.4 \mathrm{E}-13$ \\
& GOTERM_MF_FAT & Protein binding & 56.5 & $4.8 \mathrm{E}-20$ \\
& GOTERM_MF_FAT & ATP binding & 10.7 & $2.4 \mathrm{E}-5$ \\
& GOTERM_MF_FAT & Ubiquitin-protein transferase activity & 3.4 & $3.3 \mathrm{E}-6$ \\
\hline
\end{tabular}

$\mathrm{BP}$, biological process; MF, molecular function; $\mathrm{CC}$, cellular component.

GO term enrichment analysis. DEGs $(3,502)$ were then used for functional analysis via online biological classification tool DAVID (14). GO analysis was carried out which covered three aspects, named molecular function (MF), cellular component (CC) and biological process (BP) (15). The results revealed that DEGs were enriched in $873 \mathrm{BP}$ terms, $250 \mathrm{CC}$ terms and $208 \mathrm{MF}$ terms under the condition of FDR $<0.05$, $\mathrm{P}<0.05$. GO analysis results showed that upregulated DEGs were significantly enriched in biological processes (BP), including immune response, cell adhesion, cell migration and cell proliferation (Table I). The downregulated DEGs were significantly enriched in biological processes, including cell circle, spermatogenesis, and reproduction (Table I). For molecular function (MF), the upregulated DEGs were enriched in protein binding and poly(A) RNA binding, and the downregulated DEGs were enriched in protein binding and ATP binding (Table I). Besides, GO cell component (CC) analysis showed that the upregulated DEGs were significantly enriched in the cytoplasm, extracellular exosome, while downregulated DEGs were enriched in cytoplasm and nucleus (Table I).

KEGG pathway analysis. To further reveal the functions of DEGs, the DEGs with FDR $<0.05$ were entered into DAVID for KEGG pathway enrichment analysis (16). The most significantly enriched molecular pathways of the upregulated DEGs and downregulated DEGs analyzed by KEGG are showed in Table II, from which we can draw the conclusion that the upregulated DEGs were enriched in cell adhesion molecules, PI3K-AKT signaling pathway, pathways in cancer, p53 signaling pathway, and NF- $\mathrm{kB}$ signaling pathway, while the downregulated DEGs were enriched in metabolic pathway, cGMP-PKG signaling pathways, FoxO signaling pathways and Wnt signaling pathway (Table II).

PPI networks and module analysis. To analyze the interaction and acquire hub genes of potentially diagnosis-related DEGs, protein-protein interactome was constructed using STRING (17). Then we put the genes with combined score $\geq 0.7$ into Cytoscape for further analysis. The PPI network included 499 nodes and 577 interactions (Fig. 3). The top $10 \mathrm{hub}$ nodes with higher degrees were screened, including ubiquitin C (Ubc), ubiquitin B (Ubb), androgen receptor (Ar), phosphatase and tensin homolog (Pten), cyclin-dependent kinase 2 (Cdk2), cyclin-dependent kinase 4 (Cdk4), mitogenactivated protein kinase 8 (Mapk8), topoisomerase (DNA) II $\alpha$ (Top2a), cyclin-dependent kinase inhibitor 1a (Cdkn1a), ras-related C3 botulinum toxin substrate 1 (Rac1) (Fig. 3). Module genes of PPI analysis were identified by MCODE. The top 3 significant modules (Fig. 4) were selected and the functional annotation of the genes involved in the top 3 modules were analyzed by DAVID (Tables III-V), showing that module genes were related mainly to the cell cycle, cell proliferation, pathways in cancer, and PI3K-AKT signaling pathway, all of which have a close relation with tumor biology and could probably affect survival and prognosis in patients with seminoma. 


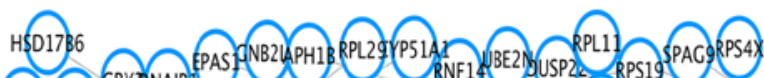

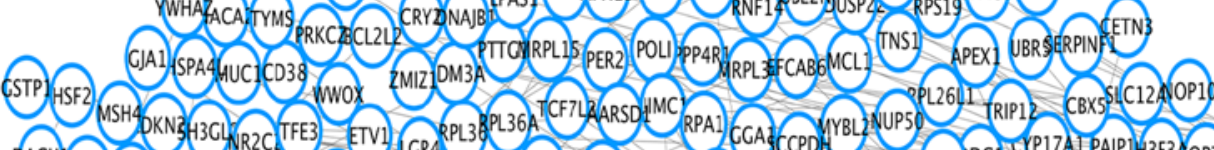

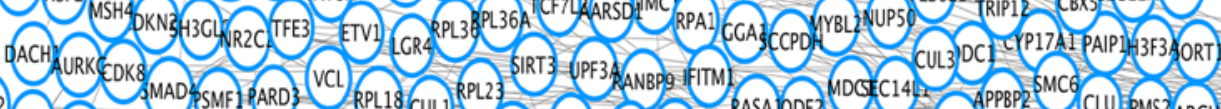

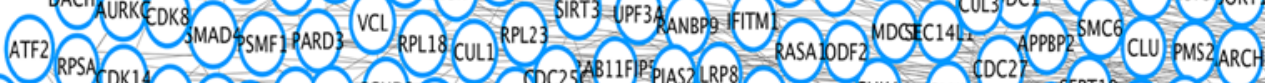

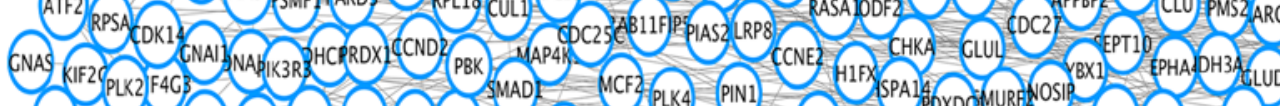

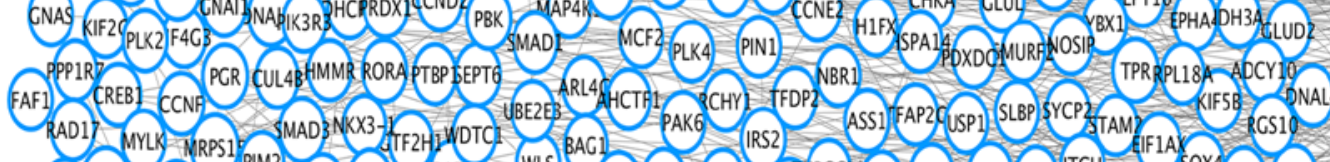

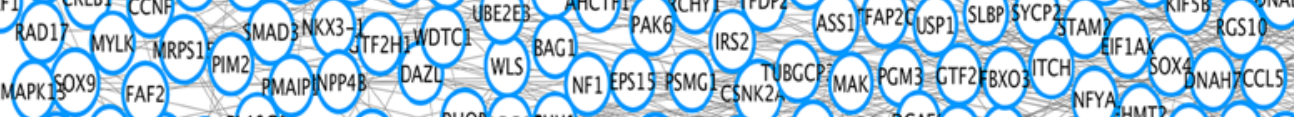

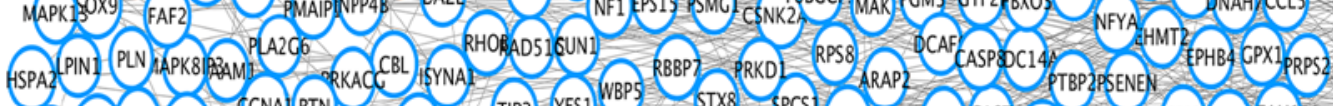

(ISPA2)

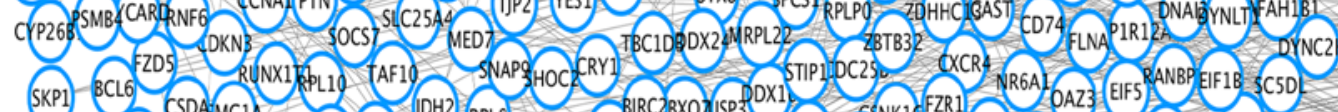

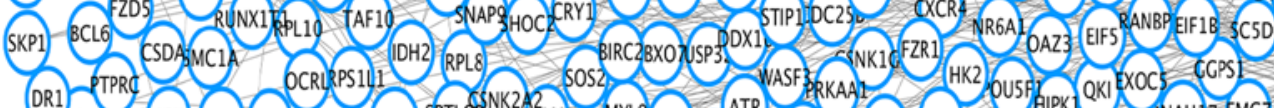

(DR1) PTPREC

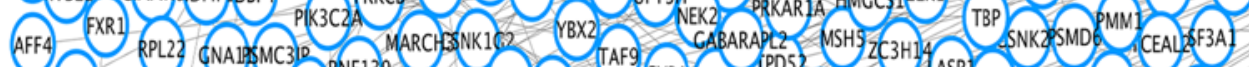

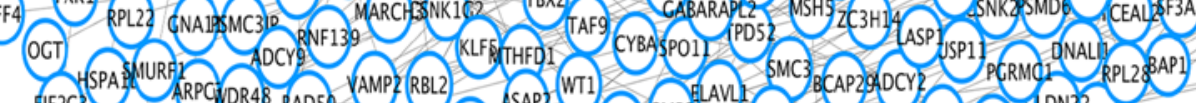

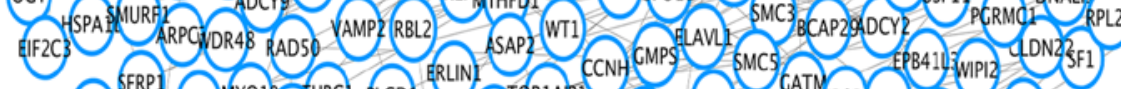

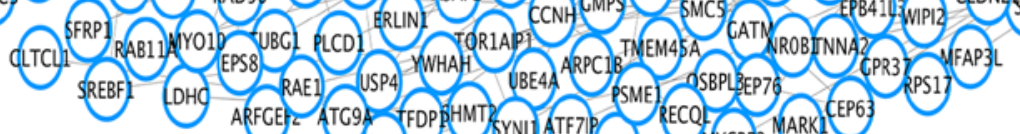

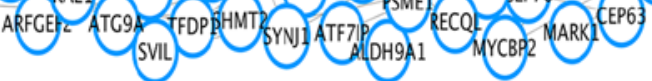

Figure 3. Protein-protein interaction networks. Red nodes, hub genes.

A

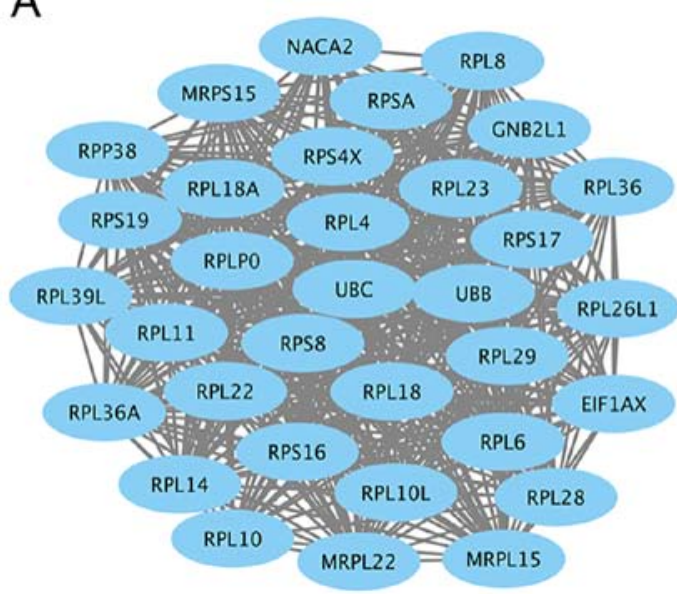

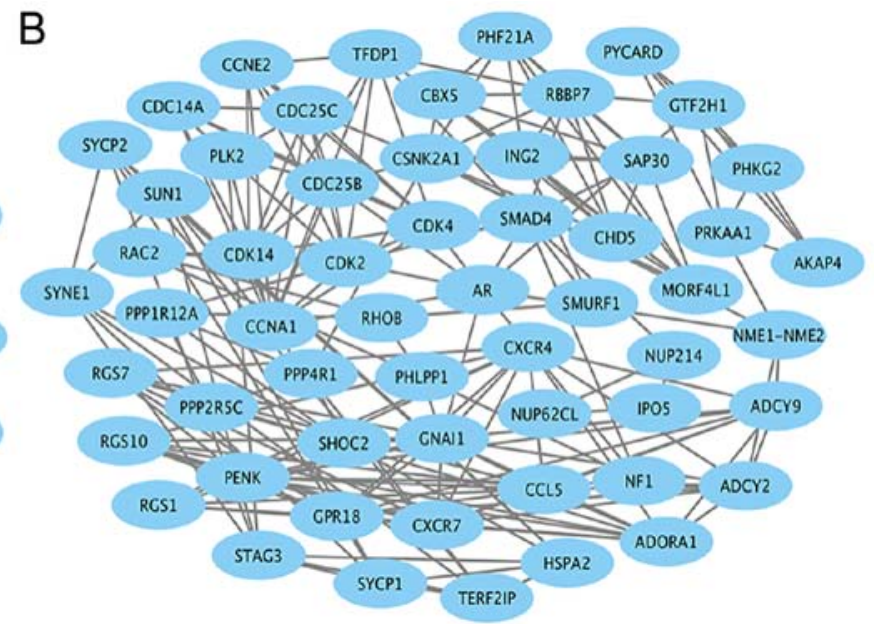

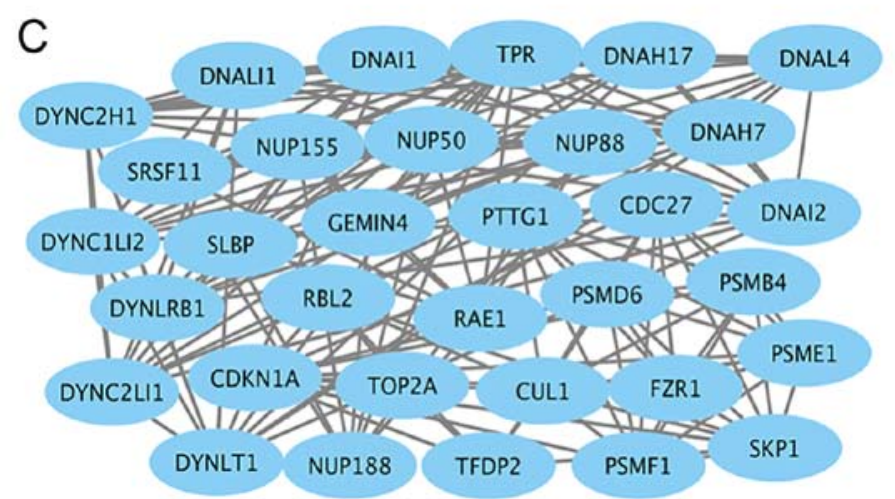

Figure 4. Top 3 modules from the protein-protein interaction network. (A) Module 1. (B) Module 2. (C) Module 3. 
Table II. KEGG pathway analysis of differentially expressed genes associated with seminoma.

\begin{tabular}{|c|c|c|c|c|}
\hline Expression & Pathway & Genes & $\begin{array}{c}\text { Gene } \\
\text { count } \%\end{array}$ & P-value \\
\hline \multirow[t]{5}{*}{ Upregulated } & $\begin{array}{l}\text { Cell adhesion } \\
\text { molecules }\end{array}$ & $\begin{array}{l}\text { CD2CD4, CD86, CD8A, CDH1, CDH3, CDH5, CLDN6, } \\
\text { CLDN7, ITGA4, SDC2, SDC3, VCAM, ITGAL, ITGAM, } \\
\text { ITGB2, ICAM1, ICAM2, ICAM3, HLA-A, HLA-B, HLA-C, } \\
\text { SELPLG, SIGLEC1, HLA-E, HLA-F, HLA-G, HLA-DMA, } \\
\text { HLA-DMB, HLA-DPA1, HLDPB1, PTPRF, SELL, } \\
\text { HLA-DQB1, HLA-DRA, HLA-DRB1, NLGN4X, NRCAM, }\end{array}$ & 3.5 & $1.9 \mathrm{E}-11$ \\
\hline & $\begin{array}{l}\text { PI3K-AKT } \\
\text { signaling pathway }\end{array}$ & $\begin{array}{l}\text { MCL1, KIT, KRAS, MYB, TCL1A, TCL1B, CREB3L2, } \\
\text { COL3A1, COL4A1, TP53, COL4A2, COL5A1, COL6A2, } \\
\text { CCND2, CCND3, CDK2, CDK4, EFNA4, EIF4B } \\
\text { EIF4EBP1, FGF13, FGFR1, FGFR2, FN1, GYS1, } \\
\text { HSP90AB1, IGF1, ITGA4 }\end{array}$ & 3.7 & $1.5 \mathrm{E}-2$ \\
\hline & Pathways in cancer & $\begin{array}{l}\text { ITGA5, IFNAR2, IL2RB, IL2RG, IL6R, LPAR6, PIK3CD, } \\
\text { RAC1, YWHAZ, BAX, CXCL12, CXCR4, CEBPA, E2F3, } \\
\text { KIT, KRAS, NFKBIA, SKP2, SMAD3, WNT2B, ADCY1, } \\
\text { ADCY7, CDH1, COL4A1, COL4A2, CSF1R, CDK2, } \\
\text { CDK4, FGF13, FGFR1, FGFR2, FN1, FZD5, HSP90AB1, } \\
\text { GF1, LPAR6, MMP2, MMP9 }\end{array}$ & 3.7 & $9.3 \mathrm{E}-2$ \\
\hline & Cell cycle & $\begin{array}{l}\text { E2F3, SKP2, SMAD3, CDC25B, CCNA2, CCND2, } \\
\text { CCND3, CDK2, CDK4, MCM2, YWHAZ, MCM3, MCM4, } \\
\text { MCM5, MCM6, MCM7, PRKDC, SFN, STAG2, SMC1A, } \\
\text { TGFB1, TP53 }\end{array}$ & 2.1 & $2.1 \mathrm{E}-4$ \\
\hline & $\begin{array}{l}\text { NF- } \kappa \mathrm{B} \text { signaling } \\
\text { pathway }\end{array}$ & $\begin{array}{l}\text { BCL2A1, CCL19, CCL4, CXCL12, CFLAR, CD14, ERC1, } \\
\text { LCK, LYN, NFKBIA, ICAM1 LAT, LY96, LTB, PLCG2, } \\
\text { PLAU, VCAM1 }\end{array}$ & 1.4 & $4.0 \mathrm{E}-3$ \\
\hline \multirow[t]{5}{*}{ Downregulated } & Metabolic pathways & $\begin{array}{l}\text { ADO, HMGCS1, HMGCS2, HIBCH, ABAT, DHCR7, ALG8, } \\
\text { ALG9, CDS1, NME5, NME7, OCRL, UAP1, ACAT1, ACO1, } \\
\text { ACSBG2, ACSL6, AK1, ADSS, ALDH1A1, ALDH1A2, } \\
\text { ALDH3A2, ALDH6A1, ALDH9A1, AGPS, ALLC, ASS1, } \\
\text { B3GALT4, BCAT1, CERS1, CHKA, CHPT1, CHPF, CKB, } \\
\text { CKM, CYP11A1 }\end{array}$ & 8 & $6.0 \mathrm{E}-4$ \\
\hline & $\begin{array}{l}\text { cGMP-PKG signaling } \\
\text { pathway }\end{array}$ & $\begin{array}{l}\text { ATP1A3, ATP2B4, GNA11, GNAI1, ATF2, ADORA1, ADCY2, } \\
\text { CREB1, CALM1, IRS2, MYL9, MYLK3MYLK, PIK3R3, } \\
\text { PLN, PPP1R12A, PPP3CA, PPP3CB, PPP3CC, SLC25A31, } \\
\text { SLC25A4 }\end{array}$ & 1.5 & $1.5 \mathrm{E}-1$ \\
\hline & Oocyte meiosis & $\begin{array}{l}\text { SKP1, ADCY2, ADCY9, AR, CALM1, CDC25C, CDC27, } \\
\text { CUL1, PTTG1, PGR, PRKACG, PPP1CC, PPP2R5C, } \\
\text { PPP2R1B, PPP3CA, PPP3CC, STAG3, SMC3, YWHAZ, }\end{array}$ & 1.4 & $1.5 \mathrm{E}-4$ \\
\hline & $\begin{array}{l}\text { FoxO signaling } \\
\text { pathway }\end{array}$ & $\begin{array}{l}\text { BRAF, BCL6, BCL2L11, GABARAP, RBL2, SMAD4, } \\
\text { CDKN1A, CDKN2D, FOXG1, IRS2, MAPK13, MAPK8, } \\
\text { PTEN, PLK2, PLK4, PRKAA1, SOS2 }\end{array}$ & 1.2 & $1.1 \mathrm{E}-2$ \\
\hline & $\begin{array}{l}\text { Wnt signaling } \\
\text { pathway }\end{array}$ & $\begin{array}{l}\text { SKP1, SMAD4, CSNK2A1, CSNK2A2, CSNK2B, CUL1, } \\
\text { DAAM1, DAAM2, LEF1, MAPK8, PRKCB, PRKACG, } \\
\text { PPP3CA, PPP3CB }\end{array}$ & 1.1 & $5.5 \mathrm{E}-2$ \\
\hline
\end{tabular}

Validation of hub genes via RT-PCR and IHC. To confirm the key genes identified by above analyses, RT-PCR assay and IHC of 5 hub genes (Ubb, Ubc, Ar, Pten and Cdk4) were conducted by using online database Oncomine and the human protein atlas. Ubb, Ubc, Ar and Pten were downregulated DEGs, while Cdk4 was an upregulated DEG. The RT-PCR results are 
Table III. Functional annotation of the significant module 1.

\begin{tabular}{lllr}
\hline Category & \multicolumn{1}{c}{ Term } & \multicolumn{1}{c}{ Gene } & P-value \\
\hline GOTERM_BP_FAT & Translation & EIF1AX, MRPL15, MRPL22, MRPS15, RPL10L, & $2.4 \mathrm{E}-33$ \\
& & RPL11, RPL14, RPL18A, RPL22, RPL23, & \\
& & RPL26L1, RPL28, RPL29, RPL36, RPL36A & \\
GOTERM_BP_FATE & RBB2 signaling pathway & UBC, UBB & $6.4 \mathrm{E}-2$ \\
KEEN PATHWAY & Ribosome & MRPL15, MRPL22, MRPS15, RPL10L, RPL11, & $2.6 \mathrm{E}-38$ \\
& & RPL14, RPL18, RPL23, RPL26, L1, RPL28, & \\
& & RPL29, RPL36, RPL36A, RPL4, RPL6, RPL8 & \\
\hline
\end{tabular}

Table IV. Functional annotation of the significant module 2.

\begin{tabular}{|c|c|c|c|}
\hline Category & Term & Gene & P-value \\
\hline GOTERM_BP_FAT & Cell cycle & $\begin{array}{l}\text { SUN1, CSNK2A1, CDC14A, CDC25B, } \\
\text { CDC25C, CCNA1, CCNE2, CDK14, } \\
\text { CDK2, CDK4, HSPA2, ING2, NUP214, } \\
\text { PLK2, PRKAA1, RHOB, STAG3 }\end{array}$ & $6.6 \mathrm{E}-10$ \\
\hline GOTERM_BP_FAT & Protein phosphorylation & $\begin{array}{l}\text { CCL5, CXCR4, NME1-NME2, PHLPP1, } \\
\text { PYCARD, SMAD4, RAC2, AR, ADORA1, } \\
\text { ADCY2, ADCY9, CDC25B, PLK2, } \\
\text { PRKAA, 1PPP4R1 }\end{array}$ & $1.3 \mathrm{E}-10$ \\
\hline GOTERM_BP_FAT & Cell proliferation & $\begin{array}{l}\text { CCL5, NME1-NME2, PYCARD, RBBP7, } \\
\text { SMAD4, ADORA1, CSNK2A1, AR, } \\
\text { CDC14A, CDC25B, CDC25C, CHD5, } \\
\text { CDK2, CDK4, ING2, NF1, PENK }\end{array}$ & $1.8 \mathrm{E}-7$ \\
\hline KEEN PATHWAY & Cell cycle & $\begin{array}{l}\text { SMAD4, CDC14A, CDC25B, CDC25C, } \\
\text { CCNA1, CCNE2, CDK2, CDK4, TFDP1 }\end{array}$ & $6.3 \mathrm{E}-8$ \\
\hline KEEN PATHWAY & Pathways in cancer & $\begin{array}{l}\text { AR, CXCR4, SMAD4, ADCY2, ADCY9, } \\
\text { CCNE2, CDK2, CDK4, RAC2, GNAI1 }\end{array}$ & $4.9 \mathrm{E}-5$ \\
\hline KEEN PATHWAY & PI3K-AKT signaling pathway & $\begin{array}{l}\text { PHLPP1, CCNE2, CDK2, CDK4, } \\
\text { PRKAA1, PPP2R5C }\end{array}$ & $2.0 \mathrm{E}-2$ \\
\hline
\end{tabular}

Table V. Functional annotation of the significant module 3.

\begin{tabular}{lllr}
\hline Category & \multicolumn{1}{c}{ Term } & \multicolumn{1}{c}{ Gene } & P-value \\
\hline GOTERM_BP_FAT & Cell cycle process & RBL2, SKP1, CDC27, CUL1, CDKN1A, DYNLT1, & 7.6E-13 \\
& & PSME1, SMB4, TOP2A, TFDP2, SLBP, TPR & \\
KEEN PATHWAY & Cell cycle & RBL2, SKP1, CDC27, CUL1, CDKN1A, PTTG1, TFDP2 & 4.0 E-6 \\
\hline
\end{tabular}

shown in Fig. 5. The relative expression levels of Cdk4 was increased, but that of Ubb, Ubc, Ar, Pten were decreased in seminoma compared with normal controls, which was consistent with the DEG analysis. Furthermore, the differences in hub gene expression levels were further conformed by IHC (Fig. 6). Moreover, we evaluated identified hub genes RNA expression by RT-qPCR in paired seminoma tissues. The result was consistent with above analysis (Fig. 7). In conclusion, our 

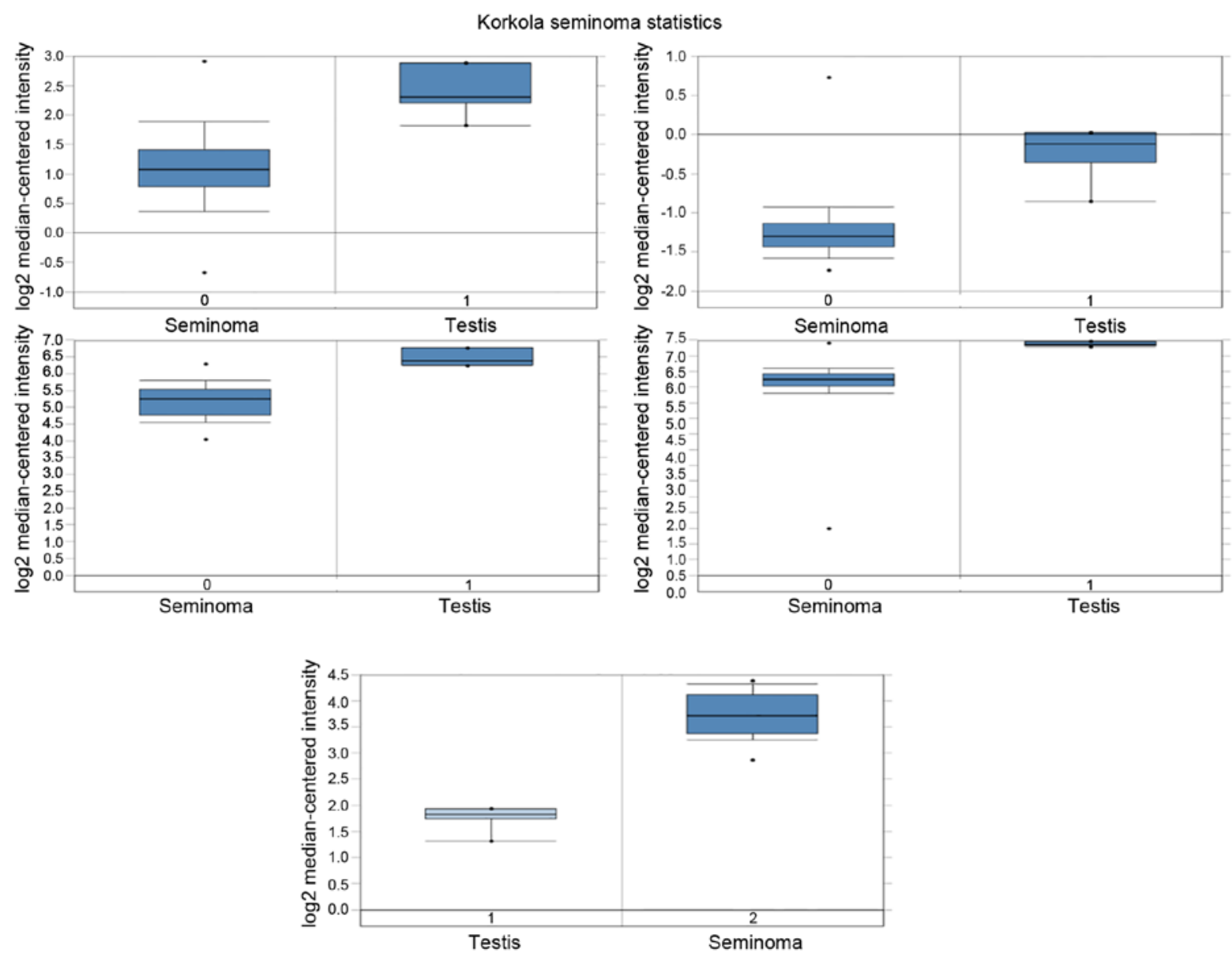

Figure 5. Top 5 hub gene mRNA level expression. PTEN, AR, UBC, UBB and CDK4.

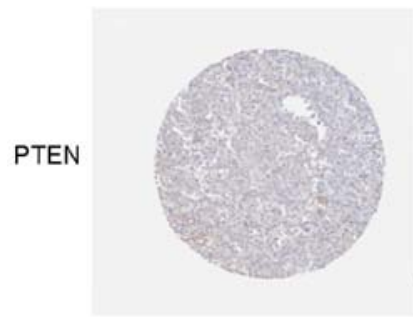

Seminoma

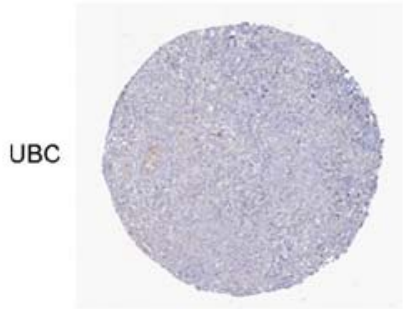

Seminoma

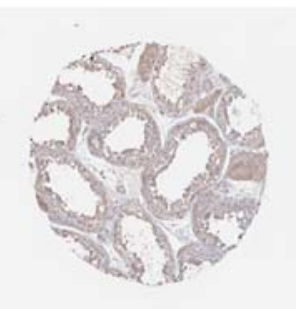

Testis

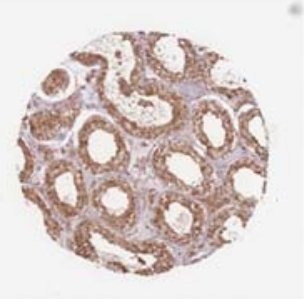

Testis

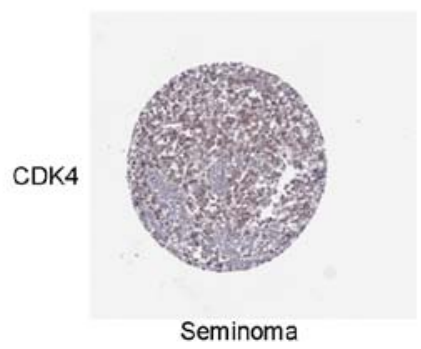

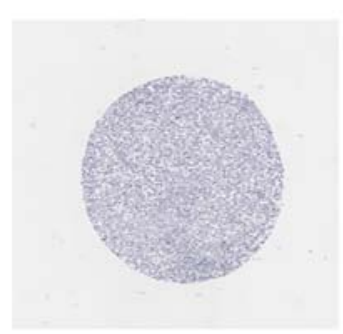

Seminoma

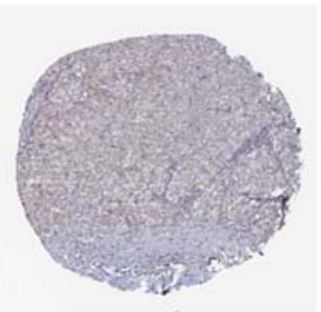

Seminoma

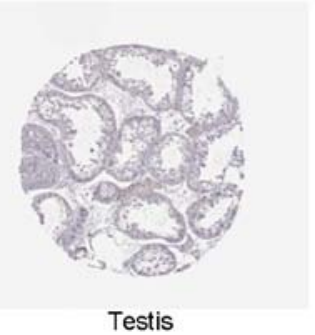

AR

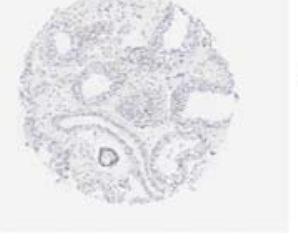

Testis

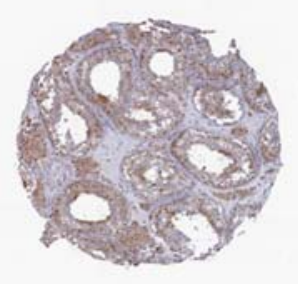

Testis

Figure 6. Top 5 hub gene protein level expression. PTEN, AR, UBC, UBB and CDK4. 
Sample 1

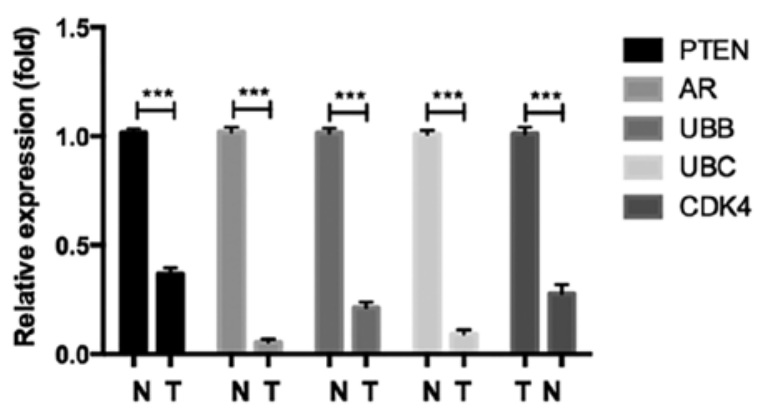

Sample 2

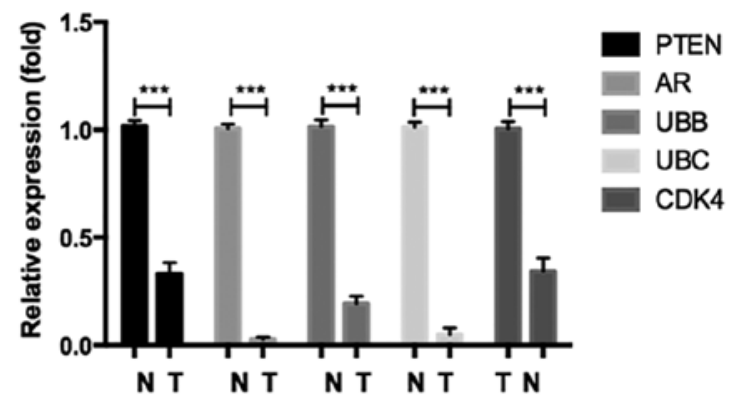

Sample 3

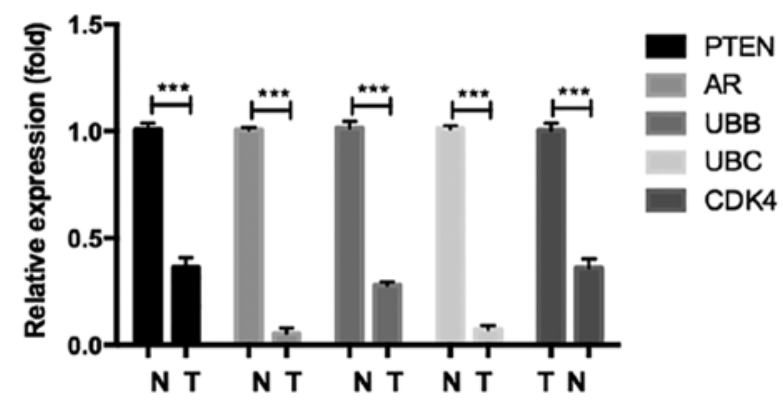

Figure 7. The expression of hub genes in clinical seminoma tissues. Relative mRNA expression level (log 2 fold change) was measured by qRT-PCR and the result of 3 samples are shown. N, normal tissues. T, tumor tissues.

results demonstrated that these hub genes were significantly differentially expressed in seminoma.

\section{Discussion}

Testicular germ cell cancer is the most common tumor among young men (aged 15-40 years) around the world (18). It is evident that there is a strong relationship between congenital anomaly (cryptorchidism) and testicular cancer (19). However, the risk factors and genetic etiology for testicular cancer are largely unknown. Consequently, understanding the molecular mechanism of TGCG is of critical importance for diagnosis and treatment. In this study, we first aimed to explore the mechanism of seminoma, which comprises $56 \%$ of total TGCGs. Nowadays, microarray and high-throughput sequencing technology give us a deep insight into the expression levels of thousands of genes in human genome simultaneously, as a result, it has been widely used to predict the potential diagnostic biomarkers and therapeutic targets for different cancers (20).

In this study, we compared gene expression profiles of seminoma tumor with normal tissues from the GEO database and identified 1,563 upregulated and 1,939 downregulated DEGs. The GO term analysis showed that upregulated DEGs were mainly composed of molecules participating in cell adhesion, PI3K-AKT signaling pathway and cell cycle. PI3K-Akt pathway is known to be a vital signaling pathway, which can promote EMT transition and have an effect on the occurrence and progression of many types of cancers $(21,22)$. The downregulated DEGs were involved in metabolic molecular pathways, cGMP-PKG signaling pathway and Oocyte meiosis. A recent study suggested cGMP-dependent protein kinase $G$ was involved in maintaining stemness of cancer stem cells and targeting this molecular pathway could effectively prevent initiation, metastasis, and relapse of the cancer (23). Hence, GO analysis revealed several possible biological processes, molecular functions and cellular components which might be involved in the initiation and development of seminoma. Function annotation and KEGG suggested that DEGs between tumor and normal controls were greater in cell cycle, pathways in cancer, cell proliferation and ERBB2 signaling pathway. It is consistent with the knowledge that defective function of cell cycle and increased cell proliferation are the main cause for cancer initiation and progression (24-26). Module analysis of the PPI network revealed that the development of seminoma was associated with cell cycle, cell proliferation and pathways in cancer, which was consistent with our pathway analysis. Some hub genes, including $U b c, U b b, M a p k 8, A r, P t e n, C d k 2$, $C d k 4, R a c l$, Top $2 a$ and $C d k n l a$ have been identified based on the degree, which can provide a new sight for the therapeutic strategy in seminoma by constructing the PPI network.

When genes such as the cell cycle regulators cyclindependent kinases $(C d k)$ mutate, they may cause cells to multiply uncontrollably growth and thus result in cancer formation. Recent studies have established cell cycle kinases as anticancer drug targets by using $C d k 4 / 6$ inhibitors $(27,28)$. Among the DEGs of seminoma in the present study, there were several genes deeply associated with the cell cycle, such as Cdk2, Cdk4, Top2a, Cdc14a, Cdc25c, Ccnal and Ccne2, indicating $C d k 2, C d k 4$ and Top $2 a$ may serve as critical genetic aberrations in seminoma.

$A r$, a downregulated DE gene, was identified as a hub gene of seminoma in this study. Androgens exert many biological effects on many tissues through the androgen 
receptor $(A r)(29,30)$. Previous studies indicated that activation of androgen/Ar signaling had a positive role on prostate cancer cell growth $(31,32)$. In the case of testes, androgen/Ar signaling was known to be indispensable for normal development and function (33). Recently, a study revealed that activation of androgen $/ A r$ signal suppressed cell growth of testicular cancer in vitro and in vivo (34). In our study, $A r$ expression was downregulated as confirmed by RT-PCR assays and IHC, indicating $A r$ is significantly negatively associated with seminoma.

Pten is a tumor suppressor and known to negatively regulate PI3K signaling to inhibit cell growth. Consequently, its suppression leads to increased proliferation and invasion ability and promotes tumorigenesis. The decreased expression of Pten is also related with the transformation of carcinoma in situ (CIS) cells into cancerous tumors $(35,36)$. Furthermore, loss of $P I K 3 I P 1$, an additional negative regulator of PIK kinase, contributed to increased relapse rate in TGCTs (37). This observation suggests the negative regulators of PI3K signaling may also be related to TGCT progression/prognosis. However, literature focused on Pten expression in seminoma is still lacking. In our study, we discovered that Pten was a downregulated DEG and significantly differently expressed based on RT-PCR assays and IHC in seminoma. These results revealed a negative role of Pten on seminoma pathogenesis, which was in accordance with previous studies.

$U b b$ and $U b c$ are members of the ubiquitin gene family. Ubiquitin is a small, highly conserved protein expressed in all eukaryotic cells, which can be covalently linked to certain target proteins to mark them for degradation by the ubiquitin-proteasome system (UPS) (38). The abundance of cellular ubiquitin is in a dynamic balance, which is ultimately maintained by de novo synthesis of ubiquitin from ubiquitin gene transcripts. Human ubiquitin is encoded by a family of multiple genes, composed of $U b a 52, U b a 80, U b b$ and $U b c$, of which $U b b$ and $U b c$ are inducible by various cell stresses (39-41). Former studies found that there are some alterations in the UB system in many types of human cancers and the deregulation of its components have been found to play key roles in cellular processes relevant to tumorigenesis $(42,43)$. Besides, reduced $U b b$ expression inhibited TSA-induced apoptotic cascade in tumor cells (38). In this study, we found the expression of $U b b$ and $U b c$ were significantly decreased in seminoma tissue via RT-PCR and IHC assay, which indicated the UB system is possibly involved in the tumorigenesis of seminoma.

In conclusion, with the microarray gene expression profiling, this study provides a comprehensive bioinformatic analysis of DEGs, which may be related to initiation and progression of seminoma. Results from our study provide a cluster of potential diagnostic-related genes and molecular pathways for future investigation and may be helpful for revealing the molecular mechanisms of seminoma. While, further molecular biological experiments are essential to demonstrate certain function of these hub genes and key molecular pathways in seminoma.

\section{References}

1. Manku G, Hueso A, Brimo F, Chan P, Gonzalez-Peramato P, Jabado N, Gayden T, Bourgey M, Riazalhosseini Y and Culty M: Changes in the expression profiles of claudins during gonocyte differentiation and in seminomas. Andrology 4: 95-110, 2016.
2. Litchfield K, Summersgill B, Yost S, Sultana R, Labreche K, Dudakia D, Renwick A, Seal S, Al-Saadi R, Broderick P, et al: Whole-exome sequencing reveals the mutational spectrum of testicular germ cell tumours. Nat Commun 6: 5973, 2015.

3. Aran V, Victorino AP, Thuler LC and Ferreira CG: Colorectal cancer: Epidemiology, disease mechanisms and interventions to reduce onset and mortality. Clin Colorectal Cancer 15: 195-203, 2016.

4. Taylor-Weiner A, Zack T, O'Donnell E, Guerriero JL, Bernard B, Reddy A, Han GC, AlDubayan S, Amin-Mansour A, Schumacher SE, et al: Genomic evolution and chemoresistance in germ-cell tumours. Nature 540: 114-118, 2016.

5. Liu T, Li R, Zhao H, Deng J, Long Y, Shuai MT, Li Q, Gu H, Chen YQ and Leng AM: eIF4E promotes tumorigenesis and modulates chemosensitivity to cisplatin in esophageal squamous cell carcinoma. Oncotarget 7: 66851-66864, 2016.

6. Chieffi P and Chieffi S: Molecular biomarkers as potential targets for therapeutic strategies in human testicular germ cell tumors: An overview. J Cell Physiol 228: 1641-1646, 2013.

7. Trabert B, Chen J, Devesa SS, Bray F and McGlynn KA: International patterns and trends in testicular cancer incidence, overall and by histologic subtype, 1973-2007. Andrology 3: 4-12, 2015.

8. Gilligan TD, Seidenfeld J, Basch EM, Einhorn LH, Fancher T, Smith DC, Stephenson AJ, Vaughn DJ, Cosby R and Hayes DF; American Society of Clinical Oncology: American Society of Clinical Oncology Clinical Practice Guideline on uses of serum tumor markers in adult males with germ cell tumors. J Clin Oncol 28: 3388-3404, 2010

9. Murray MJ, Huddart RA and Coleman N: The present and future of serum diagnostic tests for testicular germ cell tumours. Nat Rev Urol 13: 715-725, 2016.

10. Hanna NH and Einhorn LH: Testicular cancer - discoveries and updates. N Engl J Med 371: 2005-2016, 2014.

11. Sheikine Y, Genega E, Melamed J, Lee P, Reuter VE and Ye H: Molecular genetics of testicular germ cell tumors. Am J Cancer Res 2: 153-167, 2012.

12. Kanetsky PA, Mitra N, Vardhanabhuti S, Li M, Vaughn DJ, Letrero R, Ciosek SL, Doody DR, Smith LM, Weaver J, et al: Common variation in KITLG and at $5 \mathrm{q} 31.3$ predisposes to testicular germ cell cancer. Nat Genet 41: 811-815, 2009.

13. Rijlaarsdam MA and Looijenga LH: An oncofetal and developmental perspective on testicular germ cell cancer. Semin Cancer Biol 29: 59-74, 2014

14. Huang W, Sherman BT and Lempicki RA: Systematic and integrative analysis of large gene lists using DAVID bioinformatics resources. Nat Protoc 4: 44-57, 2009.

15. Consortium TGO: Gene ontology: tool for the unification of biology. The Gene Ontology Consortium. Nat Genet 25: 25-29, 2000.

16. Ogata H, Goto S, Sato K, Fujibuchi W, Bono H and Kanehisa M: KEGG: Kyoto Encyclopedia of Genes and Genomes. Nucleic Acids Res 27: 29-34, 1999.

17. Köhler S, Bauer S, Horn D and Robinson PN: Walking the interactome for prioritization of candidate disease genes. Am J Hum Genet 82: 949-958, 2008.

18. Chia VM, Quraishi SM, Devesa SS, Purdue MP, Cook MB and McGlynn KA: International trends in the incidence of testicular cancer, 1973-2002. Cancer Epidemiol Biomarkers Prev 19: 1151-1159, 2010.

19. Cook MB, Akre O, Forman D, Madigan MP, Richiardi L and McGlynn KA: A systematic review and meta-analysis of perinatal variables in relation to the risk of testicular cancer - experiences of the son. Int J Epidemiol 39: 1605-1618, 2010.

20. Peng J, Wu Y, Tian X, Pang J, Kuai L, Cao F, Qin X, Zhong J, $\mathrm{Li}$ X, Li Y, et al: High-throughput sequencing and co-expression network analysis of lncRNAs and mRNAs in early brain injury following experimental subarachnoid haemorrhage. Sci Rep 7: 46577, 2017.

21. Jain A, Tripathi R, Turpin CP, Wang $C$ and Plattner R: Abl kinase regulation by BRAF/ERK and cooperation with Akt in melanoma. Oncogene: Apr 3, 2017 (Epub ahead of print). doi: 10.1038/onc.2017.76. 2017.

22. Chen L, Fu H, Luo Y, Chen L, Cheng R, Zhang N and Guo H: cPLA2 $\alpha$ mediates TGF- $\beta$-induced epithelial-mesenchymal transition in breast cancer through PI3k/Akt signaling. Cell Death Dis 8: e2728, 2017.

23. Liu N, Mei L, Fan X, Tang C, Ji X, Hu X, Shi W, Qian Y, Hussain $\mathrm{M}, \mathrm{Wu}$ J, et al: Phosphodiesterase 5/protein kinase $\mathrm{G}$ signal governs stemness of prostate cancer stem cells through Hippo pathway. Cancer Lett 378: 38-50, 2016. 
24. Perez R, Wu N, Klipfel AA and Beart RW Jr: A better cell cycle target for gene therapy of colorectal cancer: Cyclin G. J Gastrointest Surg 7: 884-889, 2003.

25. Tominaga O1, Nita ME, Nagawa H, Fujii S, Tsuruo T and Muto T: Expressions of cell cycle regulators in human colorectal cancer cell lines. Jpn J Cancer Res 88: 855-860 1997.

26. Shen HQ, Xiao YX, She ZY, Tan FQ and Yang WX: A novel role of KIF3b in the seminoma cell cycle. Exp Cell Res 352: 95-103, 2017.

27. Sherr CJ, Beach D and Shapiro GI: Targeting CDK4 and CDK6: From discovery to therapy. Cancer Discov 6: 353-367, 2016

28. Asghar U, Witkiewicz AK, Turner NC and Knudsen ES: The history and future of targeting cyclin-dependent kinases in cancer therapy. Nat Rev Drug Discov 14: 130-146, 2015.

29. Oury F, Sumara G, Sumara O, Ferron M, Chang H, Smith CE, Hermo L, Suarez S, Roth BL, Ducy P, et al: Endocrine regulation of male fertility by the skeleton. Cell 144: 796-809, 2011.

30. Rao PM, Kelly DM and Jones TH: Testosterone and insulin resistance in the metabolic syndrome and T2DM in men. Nat Rev Endocrinol 9: 479-493, 2013

31. Qi J, Tripathi M, Mishra R, Sahgal N, Fazli L, Ettinger S, Placzek WJ, Claps G, Chung LW, Bowtell D, et al: The E3 ubiquitin ligase Siah2 contributes to castration-resistant prostate cancer by regulation of androgen receptor transcriptional activity. Cancer Cell 23: 332-346, 2013.

32. Ito S, Ueda T, Ueno A, Nakagawa $\mathrm{H}$, Taniguchi $\mathrm{H}$, Hongo $\mathrm{F}$, Kamoi K, Okihara K, Kawauchi A and Miki T: Paired box 2 upregulates androgen receptor gene expression in androgenindependent prostate cancer. FEBS J 281: 4506-4518, 2014.

33. Wang RS, Yeh S, Tzeng CR and Chang C: Androgen receptor roles in spermatogenesis and fertility: Lessons from testicular cell-specific androgen receptor knockout mice. Endocr Rev 30: 119-132, 2009.

34. Nakagawa H,Ueda T, Ito S, Shiraishi T, Taniguchi H,Kayukawa N, Nakanishi H, Ushijima S, Kanazawa M, Nakamura T, et al: Androgen suppresses testicular cancer cell growth in vitro and in vivo. Oncotarget 7: 35224-35232, 2016.
35. Di Vizio D, Cito L, Boccia A, Chieffi P, Insabato L, Pettinato G, Motti ML, Schepis F, D'Amico W, Fabiani F, et al: Loss of the tumor suppressor gene PTEN marks the transition from intratubular germ cell neoplasias (ITGCN) to invasive germ cell tumors. Oncogene 24: 1882-1894, 2005.

36. McIver SC, Stanger SJ, Santarelli DM, Roman SD, Nixon B and McLaughlin EA: A unique combination of male germ cell miRNAs coordinates gonocyte differentiation. PLoS One 7: e35553, 2012.

37. Gilbert DC, McIntyre A, Summersgill B, Missiaglia E, Goddard NC, Chandler I, Huddart RA and Shipley J: Minimum regions of genomic imbalance in stage I testicular embryonal carcinoma and association of $22 \mathrm{q}$ loss with relapse. Genes Chromosomes Cancer 50: 186-195, 2011.

38. Wu P, Tian Y, Chen G, Wang B, Gui L, Xi L, Ma X, Fang Y, Zhu T, Wang D, et al: Ubiquitin B: An essential mediator of trichostatin A-induced tumor-selective killing in human cancer cells. Cell Death Differ 17: 109-118, 2010.

39. Nenoi M: Induced accumulation of polyubiquitin gene transcripts in HeLa cells after UV-irradiation and TPA-treatment. Int J Radiat Biol 61: 205-211, 1992.

40. Finch JS, St John T, Krieg P, Bonham K, Smith HT, Fried VA and Bowden GT: Overexpression of three ubiquitin genes in mouse epidermal tumors is associated with enhanced cellular proliferation and stress. Cell Growth Differ 3: 269-278, 1992.

41. Ryu KY, Sinnar SA, Reinholdt LG, Vaccari S, Hall S, Garcia MA, Zaitseva TS, Bouley DM, Boekelheide K, Handel MA, et al: The mouse polyubiquitin gene Ubb is essential for meiotic progression. Mol Cell Biol 28: 1136-1146, 2008.

42. Ciechanover A and Schwartz AL: The ubiquitin system: Pathogenesis of human diseases and drug targeting. Biochim Biophys Acta 1695: 3-17, 2004.

43. Hoeller D and Dikic I: Targeting the ubiquitin system in cancer therapy. Nature 458: 438-444, 2009. 